\title{
Spike-timing-dependent plasticity leads to gamma band responses in a neural network
}

\author{
Ingo Fründ · Frank W. Ohl • Christoph S. Herrmann
}

Received: 25 November 2008 / Accepted: 27 August 2009 / Published online: 30 September 2009

(C) The Author(s) 2009. This article is published with open access at Springerlink.com

\begin{abstract}
Early gamma band responses of the human electroencephalogram have been identified as an early interface linking top-down and bottom-up processing. This was based on findings that observed strong sensitivity of this signal to stimulus size and at the same time, to processes of attention and memory. Here, we simulate these findings in a simple random network of biologically plausible spiking neurons. During a learning phase, different stimuli were presented to the network and the synaptic connections were modified according to a spike-timing-dependent plasticity learning rule. In a subsequent test phase, we stimulated the network with (i) patterns of different sizes to simulate bottom-up effects and (ii) with patterns that were or were not presented during the learning phase. The network displayed qualitatively similar behavior as early gamma band responses measured from the scalp of human subjects: there was a general increase in response strength with increasing stimulus size and stronger responses for learned stimuli. We demonstrated that within one neural architecture early gamma band responses can be modulated both by bottom-up factors and by basal learning mechanisms mediated via spike-timing-dependent plasticity.
\end{abstract}

I. Fründ · F. W. Ohl · C. S. Herrmann

Bernstein Group for Computational Neuroscience,

Magdeburg, Germany

I. Fründ

Technical University Berlin,

Berlin, Germany

e-mail: ingo-fruend@tu-berlin.de

F. W. Ohl

Leibniz Institute for Neurobiology, Magdeburg, Germany

e-mail: frank.ohl@ifn-magdeburg.de

C. S. Herrmann ( $\square)$

Carl-von-Ossietzky-University, Institute of Psychology,

26111 Oldenburg, Germany

e-mail: christoph.herrmann@uni-oldenburg.de
Keywords Gamma band response · Evoked - Induced · Simulation $\cdot$ Spiking neuron

\section{Introduction}

The brain is continuously active. This spontaneously emerging activity manifests itself on different scales ranging from spontaneous spiking of single cells (Abeles 1991), over finegrained spatio-temporal patterns as revealed by optical imaging (Arieli et al. 1996; Kenet et al. 2003; Tsodyks et al. 1999) to large-scale brain rhythms (Başar 1980; Berger 1929), and coordinated fluctuations of local brain metabolism (Fox et al. 2007). In many cases, responses to specific stimuli emerge from this ongoing activity in the form of transient resets (Fründ et al. 2007b; Hanslmayr et al. 2007; Makeig et al. 2002).

A particular type of such transient responses is the early gamma band response (Herrmann et al. 2004b; Karakaş and Başar 1998; Tiitinen et al. 1993). This response occurs at roughly $30 \mathrm{~Hz}$ at a latency of approximately $90 \mathrm{~ms}$ with respect to a visual stimulus in stimulus-locked averages. The visually evoked early gamma band response is strongest for large, centrally presented stimuli (Busch et al. 2004) with low spatial frequencies (Fründ et al. 2007a) and high contrast (Schadow et al. 2007b). Despite its relatively short latency, the early gamma band response is modulated by attention (Busch et al. 2006b; Debener et al. 2003; Tiitinen et al. 1993) and by similarity between stimulus and patterns stored in long-term memory (Fründ et al. 2008a; Herrmann et al. 2004a; Morup et al. 2006).

Gamma band responses have also been reported for a later time interval around 200 and $300 \mathrm{~ms}$ after stimulus onset (e.g., Tallon-Baudry and Bertrand 1999; Gruber and Müller 2006; Busch et al. 2006a; Lachaux et al. 2005). These later, 


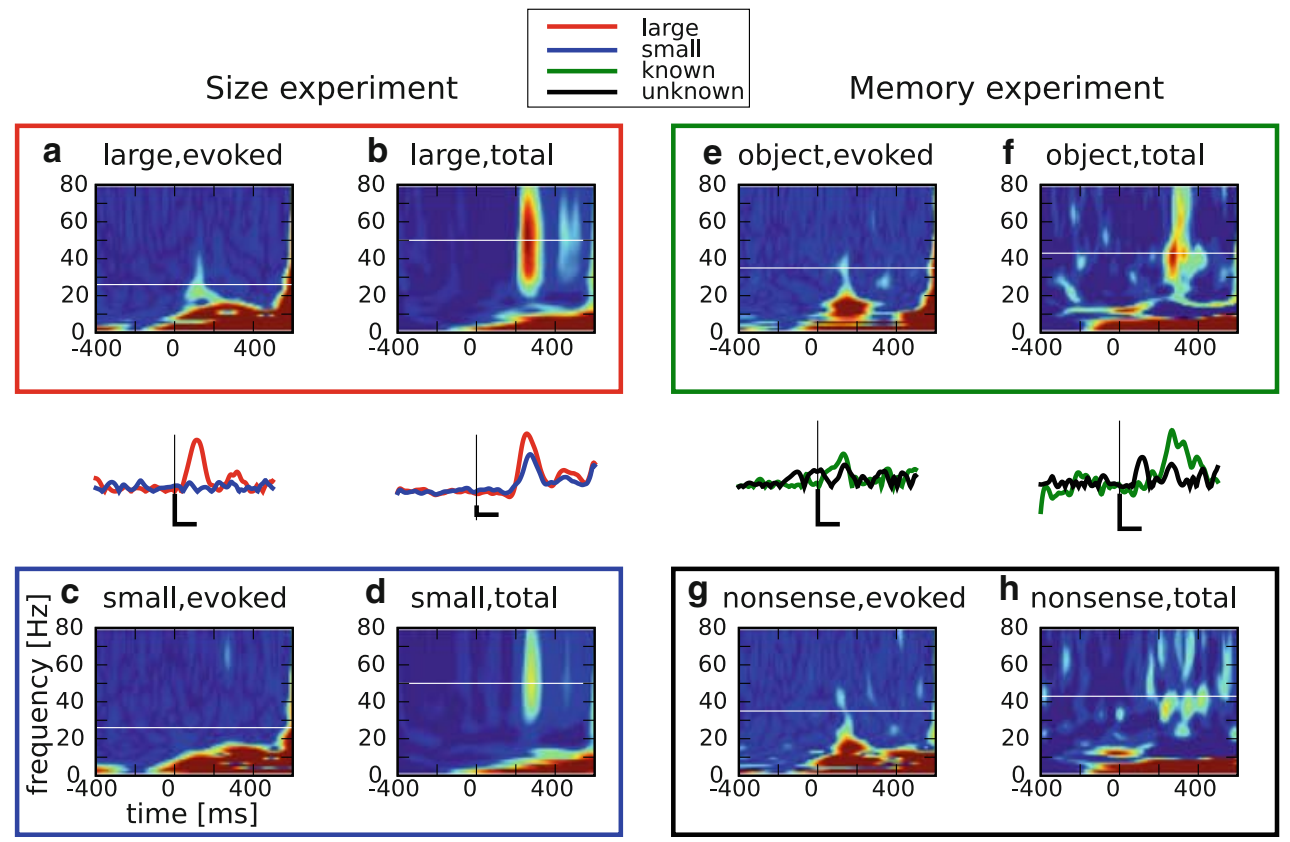

Fig. 1 Summary of experimentally obtained results about event related gamma band responses. Left side: single participant taken from the size experiment in Fründ et al. (2008b). Right side: single participant taken from the memory experiment in Fründ et al. (2008a). a-h Time-frequency plots of activity. abscissa is time in ms, ordinate is frequency in Hz. Amplitudes are coded in color: high amplitudes correspond to reddish colors, low amplitudes correspond to blueish colors. a Evoked response to large stimuli, b total amplitude response for large stimuli, $\mathbf{c}$ evoked response to small stimuli, $\mathbf{d}$ total amplitude response for small stimuli, e evoked response to known stimuli, $\mathbf{f}$ total amplitude response

for known stimuli, $\mathbf{g}$ evoked response to unknown stimuli, $\mathbf{h}$ total amplitude response for unknown stimuli. In the middle row, the time courses of activity for the respective conditions is illustrated. The scale bars correspond to $100 \mathrm{~ms}$ horizontal and $0.1 \mu \mathrm{V}$ vertical. The frequency at which the time course data were determined is marked as a white line in the time-frequency plots $(\mathbf{a}-\mathbf{h})$. Absolute amplitudes of the responses depend on various aspects of data recording and processing and differ considerably between the two experiments. They are omitted here for clarity

so-called induced gamma band responses (Basar-Eroglu et al. 1996), are less strictly locked to the stimulus and are usually observed in the form of later amplitude modulations of the activity.

Here, we ask, how in particular early gamma band responses can emerge from the background of spontaneous activity. We simulated a sparsely connected network of spiking neurons. After the network had been trained for $24 \mathrm{~h}$ using spike-timing-dependent plasticity (Bi and Poo 1998; Markram et al. 1997), it was tested with a series of input patterns. We observed similar patterns as in human participants (see Fig. 1 for a summary of the results obtained with human participants). Early gamma band responses were stronger for large stimuli. In addition, early gamma band responses were augmented for stimuli that had been repeatedly presented during the training phase. In addition, later "induced" gamma band responses were found, too.

\section{Simulation}

We simulated a highly simplified network of spiking neurons. This network represents a small group of neurons in

an early sensory area. The EEG data, we compare with the model, are from visual experiments. Although similar mechanisms might presumably be valid for the auditory modality, too, we presently think of the network as residing in primary or early secondary visual cortex. The network was in large part adapted from Izhikevich (2006). The implementation by Izhikevich (2006) was altered to facilitate storage of networks, separate training and testing sessions, and to enable stimulation of selected cells within the network. In the following, we present details about the simulation.

\subsection{Single neuron model}

We simulated single neurons using a generalized quadratic integrate-and-fire neuron as proposed by Izhikevich (2003). This model is characterized by two ordinary differential equations

$\dot{v}=0.04 v^{2}+5 v+140-u+I, \quad \dot{u}=a(b v-u)$,

and an auxiliary after-spike resetting, if $v \geq 30 \mathrm{mV}, v$ is reset to a value $c$, and $u$ is incremented by a value $d$. Here, $v$ and $u$ are dimensionless variables, $a, b, c$, and $d$ are dimensionless real parameters, and $\dot{x}:=\mathrm{d} x / \mathrm{d} t$, where $t$ is time. 
The variable $v$ can be interpreted as the membrane potential and $u$ represents a membrane recovery variable that accounts for the activation of $\mathrm{K}^{+}$ionic currents and inactivation of $\mathrm{Na}^{+}$ionic currents. All inputs into the cell (synaptic $I_{\text {syn }}$ as well as external $I_{\text {ext }}$ ) are collapsed into the dimensionless variable $I:=I_{\text {syn }}+I_{\text {ext }}$.

The network consisted of 800 excitatory cells and 200 inhibitory cells. The parameters $a, b, c$, and $d$ were tuned in order to make the excitatory cells resemble regular spiking cells (RS, Connors and Gutnick 1990), and inhibitory cells resemble fast spiking cells (FS, Connors and Gutnick 1990). These parameters are described in more detail by Izhikevich (2003).

\subsection{Network connections}

Each neuron in the network projected to a total of 100 randomly chosen other neurons in the network (equal probability for each neuron). Thus $10 \%$ of all possible connections were realized. Neurons never projected to themselves, and inhibitory cells only projected to excitatory cells. If a presynaptic neuron $i$ fired a spike at time $t_{i}^{(k)}$, the membrane potential of the postsynaptic neuron $j$ received an impulse of strength $s_{i j}$ after a delay at time $t^{(k)}+\Delta t$. Thus, in total the synaptic current $I_{\text {syn }}^{(j)}$ of a postsynaptic neuron $j$ could be written

$I_{\mathrm{syn}}^{(j)}(t)=\sum_{k: t_{i}^{(k)}<t} \sum_{\text {projects to } j} s_{i j} \delta\left(t-t_{i}^{(k)}-\Delta t\right)$.

The synaptic delay $\Delta t$ differed between synapses. Delays of excitatory synapses were uniformly distributed between 0 (instantaneous) and $20 \mathrm{~ms}$. Delays of inhibitory synapses were fixed at $0 \mathrm{~ms}$. At the beginning of the simulation the synaptic strength of each excitatory synapse was set to $s=6$, and the synaptic strength of each inhibitory synapse was set to $s=-5$. These synaptic strengths correspond to postsynaptic potentials of $\approx 5.5 \mathrm{mV}(s=6)$ and $\approx 4.3 \mathrm{mV}(s=-5)$. The exact size of the postsynaptic potential depended on the state of the neuron before an action potential arrived. The values above are averages derived from simulations in which single neurons were first driven to a steady state and then received a pulse. The amplitude of the postsynaptic potential depended on both, the steady state and the strength of the pulse. Synaptic strengths were chosen relatively high compared to typical physiological values to compensate for the small size of the network with few inputs arriving at each cell.

In an initial training phase, the network synapses were modified by spike-timing-dependent plasticity. Each spike that arrives from an excitatory presynaptic neuron at a postsynaptic neuron, might trigger a spike in the postsynaptic neuron. Therefore, the corresponding synapse is strengthened after each spike. In addition, each spike that arrives right after the postsynaptic neuron fired, weakens the respective synapse. Details of the implementation are reported by Izhikevich (2006). The initial training phase consisted of $24 \mathrm{~h}$ simulated time. During the training phase, one of two stimuli was presented to the network every second. Both stimuli excited disjunct subsets of 50 of the excitatory neurons. Stimuli consisted of a rectangular current of strength 3.5. These stimuli were presented for $300 \mathrm{~ms}$ with a fixed inter-stimulus interval of $1 \mathrm{~s}$. After the initial training phase, learning was disabled and a number of different experiments were performed on the trained network. Learning was disabled, in order to avoid effects due to the sequence of the experiments and the exact sequence of the stimuli within an experiment.

\subsection{Simulated experiments}

Two types of experiments were performed with the trained network. The first of the two experiments probed for stimulus-driven responses in the trained network. The second experiment probed for memory effects in the trained network.

The first simulation experiment resembled the EEG experiments by Busch et al. (2004) and Fründ et al. (2008b). Human EEG data from the latter experiment are presented in Fig. 1 (left). The trained network was stimulated with stimuli of three different sizes. In the visual system, the size of the excited cortical area is a monotonic function of the retinal size of a stimulus for centrally presented stimuli. Thus, we modeled stimulus size as the number of cells that were excited by a stimulus. Stimuli were presented to 20,50 , or 100 cells, corresponding to a small, medium, or large stimulus. The stimulus consisted of a rectangular current of strength 3.5 that was injected into the cell for $300 \mathrm{~ms}$. Each stimulus level was presented 100 times. The stimulus did not overlap with the cells that were directly stimulated during learning. In order to simulate the variability across human participants and to compute statistical parameters, the experiment was repeated 13 times and the network was retrained for each repetition.

The second simulation experiment resembled the EEG experiments by Herrmann et al. (2004a) and Fründ et al. (2008a). Human EEG data from the latter experiment are presented in Fig. 1 (right). During the training phase, two stimuli were repeatedly presented to the network. Thus, during training, network connections were adapted to these stimuli. After training, these stimuli were considered "known". To simulate a memory experiment similar to the ones by Herrmann et al. (2004a) and Fründ et al. (2008a), the trained network was stimulated with the same stimuli that were used during the training phase. In addition, two new stimuli were presented to the network that had not been presented during the training phase. Each stimulus was presented 100 times. 
The experiment was repeated 13 times and the network was retrained for each repetition.

\subsection{Data postprocessing}

The experiments that have been simulated in the present study were confined by their technical approach to mass action of neural activity (Freeman 1975, 2000). This observable is derived from superimposed postsynaptic potentials of large numbers of single neurons. Postsynaptic potentials are not directly accessible from the model presented here. There is no satisfactory way how to separate postsynaptic potentials from membrane potentials. Any kind of low pass filtering would introduce ringing or other types of artifacts. It might be argued that each spike triggers a postsynaptic potential and thus, the number of spikes should be proportional. However, this is only approximately true. If a cell has more than one connection to other cells, its spikes should be weighted depending on that and on the respective connection weights. Thus, the generation between single-cell activity would change during learning. This introduces room for a lot of ambiguity. We selected another approach. We simply averaged the membrane potential across all cells in the network. This does weight a cells spikes depending on their impact on target cells. In addition, it does not introduce any filtering artifacts. We will refer to this average membrane potential as a simulated local field potential (LFP).

We studied early gamma band responses in the simulated LFP. As early gamma band responses in humans are defined by (i) timing (60-120 ms poststimulus) and (ii) frequency $(\approx 25 \mathrm{~Hz}$ and higher), we derived time frequency representations of the simulated LFP. These time frequency representations were based on a wavelet transform with a complex morlet wavelet. The morlet wavelets were approximated by modulated gaussians, that were scaled to the desired frequency, and evaluated on a grid that spanned 6 cycles of the wavelet's center frequency. At $1 \mathrm{~Hz}$, the wavelet had the form

$\Psi(t) \propto \exp \left(-\frac{t^{2}}{2}\right) \exp (2 \pi \imath t)$,

with $l=\sqrt{-1}$. The wavelet transform of a signal $x(t)$ at scale $a=f^{-1}$ was then given as the convolution with the scaled wavelet

$L_{\Psi} x(t, a)=\left(x * \Psi_{a}\right)(t), \quad \Psi_{a}(\cdot)=\frac{1}{a} \Psi\left(\frac{\cdot}{a}\right)$,

where $*$ denotes complex convolution. The wavelet transform was evaluated at frequencies ranging from 1 to $80 \mathrm{~Hz}$ in $1 \mathrm{~Hz}$ steps.

After wavelet transformation of the data three statistical quantities were derived. First, we averaged the population average of the membrane potential time courses from all pre- sentations of the same stimulus. We applied the wavelet transform to the average time course, and considered only the absolute value of the result. This quantity will be referred to as evoked activity. The evoked activity identifies all components of the response that are time locked to the onset of a stimulus. Second, we applied the wavelet transform to the population average of the membrane potential at each stimulus presentation. These data were then normalized to have unit amplitude, and were subsequently averaged. This results in a time-frequency representation of a quantity that is known as mean resultant length in statistics (Fisher 1993). In the EEG literature, this quantity is known as the phase-locking factor (PLF, Tallon-Baudry et al. 1996). We will use the second term because this quantity is 1 for a situation in which all the individual time courses are strictly phase-locked to the stimulus. The PLF approaches zero for constellations in which the phases of the individual time courses have random phase in the sense that they cancel out in the average. As a third quantity, we derived a time-frequency representation of the average amplitude of the individual time courses of population average of the membrane potential. We applied the wavelet transform to the simulated LFP at each stimulus presentation and averaged the absolute values of these wavelet transformed data.

Evoked activity is derived from an average across trials. An increase in evoked activity can result from two mechanisms: Either a response component could be enhanced or the temporal alignment of a response component across trials could become stricter. Clearly both of these mechanisms could interact, and actually they do so in real EEG data (see, Busch et al. 2006b, for an example). Evoked activity alone cannot differentiate between these two alternatives. However, taking into account PLF and the average amplitudes of the individual time courses can do this. Enhancement of a response component without accompanying temporal alignment change should result in a selective increase of the average amplitudes. In contrast, increased temporal alignment across trials should result in a selective increase of the PLF. The latter mechanism has been reported to be dominant for early gamma band responses (e.g., Fründ et al. 2007b).

Thus, we described network activity with respect to the stimulus in terms of three quantities: evoked activity, the PLF, and the average amplitude.

Data from the two experiments were statistically analyzed by means of an analysis of variance (ANOVA). The dependent variable for the ANOVA was the average evoked activity in a time range from 0 to $100 \mathrm{~ms}$ after stimulus onset. Two ANOVAs were performed: one on the data from the size experiment with a three level factor SIZE, and one on the data from the memory experiment with a two level factor FAMILIARITY.

We observed two prominent response components: one early component that was phase-locked to the onset of the 
stimulus and a late component that was an amplitude increase without phase-locking to the stimulus. To quantify the contributions of single neurons to each of these response components, patterns were derived for each of these components. The correlations between the pattern and the activity of each single neuron were considered a measure of the contribution of this neuron to the respective response component.

The early component was most prominent in time-frequency representations of the event-related potential, i.e., the average across all trials. To obtain a pattern for this response, we limited the bandwidth of the event-related potential to frequencies between 18 and $32 \mathrm{~Hz}$ (128 point digital bandpass filter with $5 \mathrm{~Hz}$-wide transition bands). The pattern was then defined as the time course of this band-limited signal in a temporal window from $25 \mathrm{~ms}$ before stimulus onset to $100 \mathrm{~ms}$ after stimulus onset. The time course of each neuron on each trial was compared to this pattern. The outcome of each comparison was quantified by a linear correlation. This way we obtained one correlation for each trial and each neuron.

The late component was most prominent in time-frequency representations of the average amplitude across trials. This indicated that the phase of the contributions to this response varies from trial to trial, although the timing as well as the frequency range remained relatively constant. It is therefore not possible to quantify the contributions of single cells to this response as the correlations with a single pattern. However, a necessary condition for the contribution of a particular cell is easily formulated: if a cell contributes to the late increase in amplitudes, it has to contribute to the simulated LFP on each single trial in the same time and frequency range. Thus, we limited the bandwidth of the averaged membrane potential on each single trial to frequencies between 50 and $70 \mathrm{~Hz}$ (164 point digital bandpass filter with $10 \mathrm{~Hz}$-wide transition bands) and selected the time course from 100 to $500 \mathrm{~ms}$ as the pattern for that particular trial. For each trial, we constructed a pattern for the late gamma band response and calculated the correlation between this pattern and the time course of the membrane potential of each single cell on that trial. This resulted in one correlation for each cell and each trial.

\section{Results}

\subsection{Network training}

In Fig. 2 the evolution of network activity during the training phase of the network is illustrated. During an initial phase (Fig. 2a), the network displayed global, burst-like activity. The inter-burst frequency was approximately $4 \mathrm{~Hz}$. During this phase, the network did virtually not respond to the stimulus that was applied to the first 50 cells. During training, the slow, global activity diminished and a characteristic response pattern emerged. Figure $2 b$ displays the correlation matrix of the responses in part (a). After a short transitional phase, all responses were highly correlated. To estimate the number of different dynamical patterns that dominated the learning dynamics, we performed an eigenvalue analysis (sometimes also known as principal component analysis). Figure $2 \mathrm{~d}$ displays the eigenvalues of the correlation matrix. The eigenvalues indicate how much of the correlation matrix can be explained by the corresponding eigenvector. The eigenvalue spectrum decreases very rapidly. Only the first two eigenvalues are visibly different from zero. The eigenvalues indicate that the first two eigenvectors capture most of the dynamics of the responses during learning. The eigenvectors that correspond to these two eigenvalues are displayed in Fig. 2e. The first eigenvector (blue) starts close to zero and reaches a stable plateau during the first 2 or $3 \mathrm{~h}$ of training. The second eigenvector (red) starts with a relatively high value and decays rapidly to zero. We can reconstruct the time course of the response pattern that is associated with one of these eigenvectors by calculating a weighted sum of the hour by hour network responses weighted by the eigenvector elements. These reconstructed response patterns are displayed in Fig. 2 (f: first eigenvector, g: second eigenvector). The response pattern associated with the first eigenvector is very similar to the network response that emerges during learning. The response pattern associated with the second eigenvector resembles the network response at the beginning of the training period. Figure $2 \mathrm{c}$ illustrates the emergence of the evoked network response. This response starts at a value close to zero and rises rapidly to a relatively stable plateau. We fitted the data with an exponential function $\mathrm{ERP}=a(1-\exp (-\tau t))$ to determine the rate $\tau$ of decay toward the plateau $a$. The rate of decay toward the plateau was $\tau=2.01 \mathrm{~h}^{-1}$. The time course of the evoked network response is well represented by the first eigenvector of the correlation matrix (albeit with inverse sign, also compare with Fig. 2f). We repeated the same analysis using network tests every $15 \mathrm{~min}$. In this case, the same observations were made. In particular, the first eigenvector highly resembled an evoked gamma band response. However, the rate of decay toward the plateau was faster in this case $\left(\tau=4.60 \mathrm{~h}^{-1}\right)$.

Similar to the network responses, the synaptic weights initially underwent large changes (Fig. 2h,i). Although synaptic weights were not fixed after the first 2 or $3 \mathrm{~h}$, the overall pattern remained stable (Fig. 2h; note that synapses 80,000 100,000 were inhibitory and remained fixed throughout the simulation) and the average synaptic strength did not change much (Fig. 2).

Training altered the topology of the networks. During the training period a snapshot of the network was stored every hour. Figure 3 a shows the differences in network connectivity between successive hours of training. Initially, these differences had relatively large amplitudes. This indicates that the 
(a) ERPs

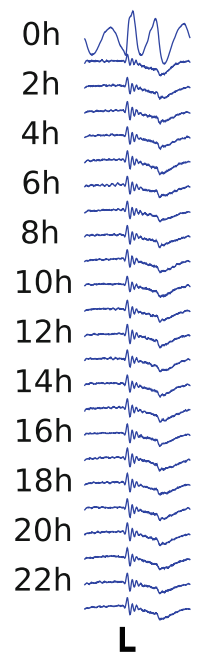

(b) correlations
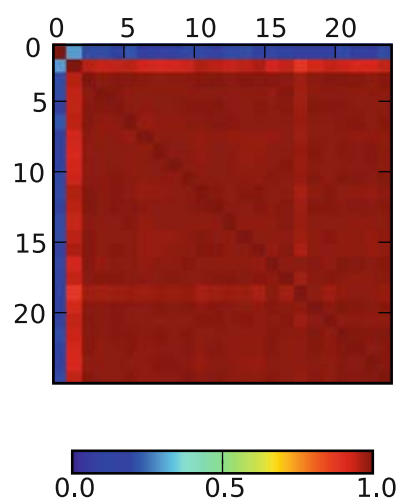

(f)

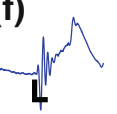

(g)

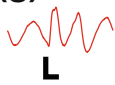

(h) time evolution of synapse strengths

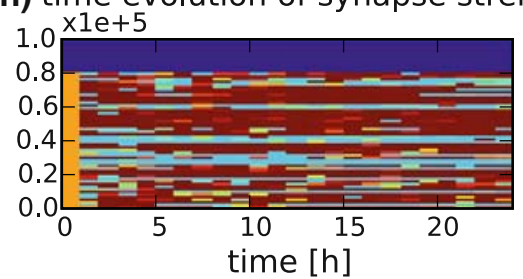

Fig. 2 Event-related neural dynamics during learning. a Event-related potential derived from 100 stimulus presentations during training. Initially, the network was engaged in slow oscillations with high amplitude and did not display a clear response. After $1 \mathrm{~h}$ of training, a characteristic response pattern started to emerge that stabilized within $2 \mathrm{~h}$. The scale bars correspond to $100 \mathrm{~ms}$ horizontal and to an arbitrary amplitude of 0.2 vertical. The vertical part of the scale bar is aligned with the onset of the stimulus. b Correlation matrix of the correlation between the event-related potentials from part (a). The color of point $(x, y)$ in the matrix displays the correlation between the event-related potential after $x$ hours with the event-related potential after $y$ hours. $\mathbf{c}$ Enhancement of the event-related potential with respect to prestimulus standard deviation during the training phase. A stable response emerged during the first 2 or $3 \mathrm{~h}$ that subsequently fluctuated around a fixed response enhancement. The solid line indicates a least-squares fit of the expression $\mathrm{ERP}=a(1-\exp (-\tau t))$ to the data. The fitted parameters were:

network was largely restructured during these initial stages of learning. After 2 or $3 \mathrm{~h}$, when the network had reached its steady state, the network connections became more stable. However, instead of remaining in a fixed state, the network connections remained changing constantly. This was in contrast with the very stable responses we observed in Fig. 2. To summarize the total effect of training, we averaged the synaptic output for each presynaptic neuron (see Fig. 3b; note that cells 800-1,000 are inhibitory). Before training, the averaged synaptic output for each presynaptic neuron was fixed (blue line in Fig. 3b). For most neurons, the

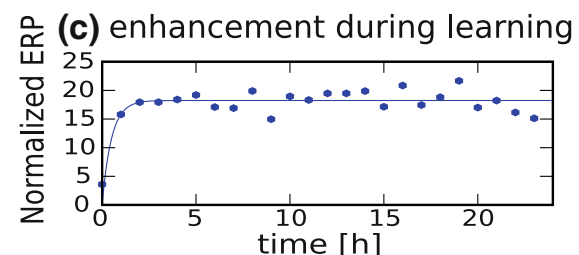

(d) eigenvalues of ERP

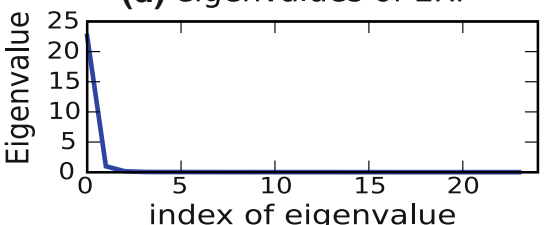

(e) eigenvectors of ERP

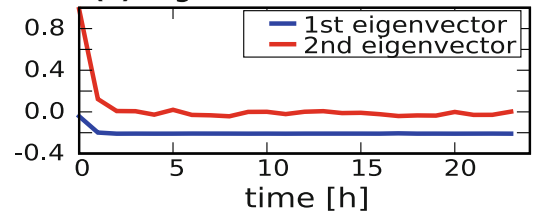

(i) average synapse strength

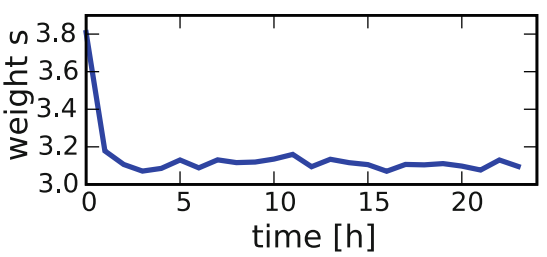

asymptotic normalized response $a=18.24$ and time constant of learning $\tau=2.01 \mathrm{~h}^{-1}$. d Eigenvalues of the correlation matrix in (b). Only two eigenvalues seem to be sufficient to describe the largest part of the correlation matrix. e The first two eigenvectors of the correlation matrix in (b). The first eigenvector (blue) starts close to zero and reaches a stable plateau during the first 2 or $3 \mathrm{~h}$ of training. The second eigenvector ( $r e d$ ) starts with a relatively high value and decays rapidly to zero. $\mathbf{f}$ Time course of the first eigenvector. Scale bars are like in (a). g Time course of the second eigenvector. Scale bars are like in (a). h Time evolution of synapse strengths during learning. After an initial strong change, a stable pattern emerges. Note that synapses with indices ranging from 80,000 to 100,000 were inhibitory and remained fixed throughout the simulation. i Average strength of synapses in the network. During the first $2 \mathrm{~h}$ of learning the synapse strengths were down-regulated and remained stable, on average, afterward

synaptic output decreased during training (see green line in Fig. 3b). The average output increased for only very few neurons. These neurons were unsystematically distributed across the whole excitatory population. The network before training and the network after training are displayed in Fig. $3 \mathrm{c}$ and d, respectively. Although the overall structure of these networks looked similar, the excitatory connections in the trained network seemed to be reduced. This was particularly true for connections from excitatory neurons to excitatory neurons. Figure $3 \mathrm{f}$ confirms this view. Here, the average input into each neuron is plotted. The blue line indicates the network before 
(a) changes of synaptic weights during training
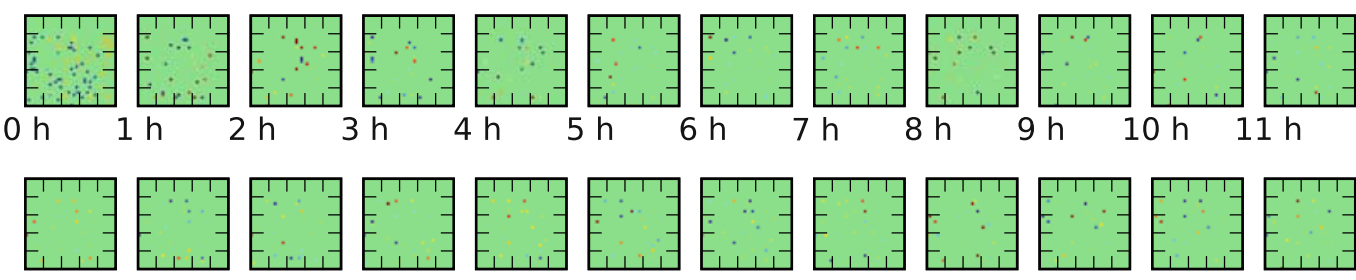

$7 \mathrm{~h} \quad 8 \mathrm{~h}$

$9 \mathrm{~h} \quad 10 \mathrm{~h} \quad 11 \mathrm{~h}$

$12 \mathrm{~h} \quad 13 \mathrm{~h}$

$14 \mathrm{~h}$
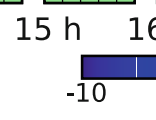

$17 \mathrm{~h} \quad 18 \mathrm{~h}$
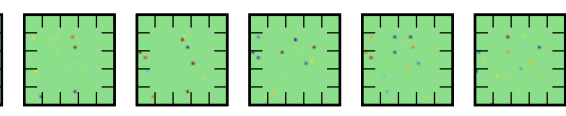

$-10$

$-3$

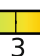

$21 \mathrm{~h} \quad 22 \mathrm{~h} \quad 23 \mathrm{~h}$

(b) output

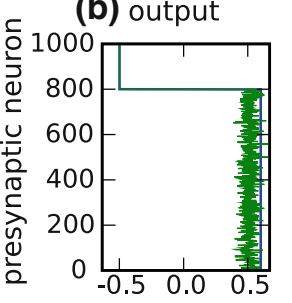

average strength

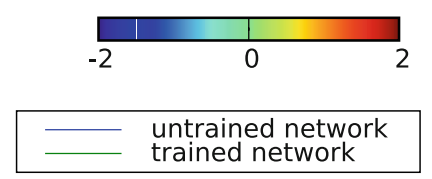

(c) untrained net

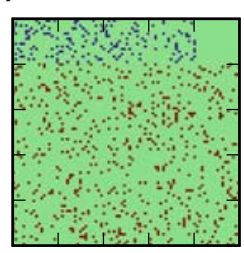

(d) trained net
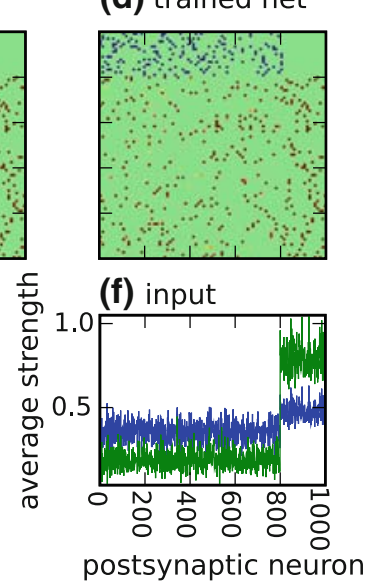

(e) sparseness of networks
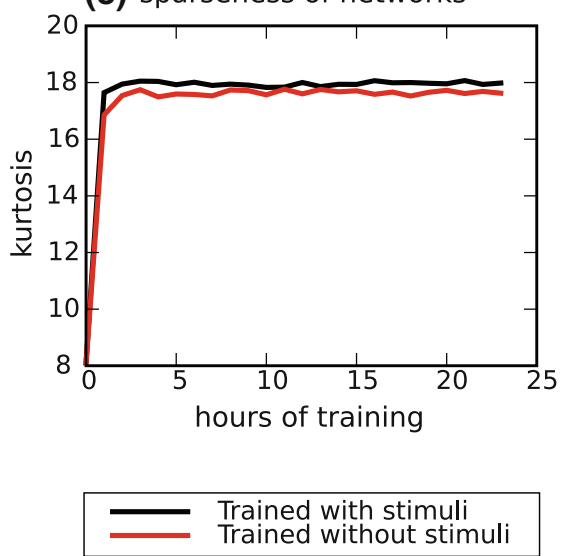

Fig. 3 Training effects on network topology. a Differences between synapse strengths between successive training hours. Initially many synapses were modified. After the network had reached its stable state in hour 2 or 3 , the synapses did not remain static but rather kept changing although with lower amplitude of the changes. b Averaged output of cells in the network. Blue line: network before training, green line: network after $24 \mathrm{~h}$ of training. Note that the blue line is largely hidden by the green line. Further note that the main effect of training is mainly to weaken excitation in the network. $\mathbf{c}$ Network topology prior to training. $y$-axis is neuron index as in (b), $x$-axis is neuron index as in (f). Note that cells 800-1000 are inhibitory. d Network topology after training. Axes like in (c). Note that the main effect of training is to increase the sparseness of the excitatory connections. e Evolution of sparseness of network connections. Sparseness initially increases rapidly and reaches a stable plateau after 2 or $3 \mathrm{~h}$ of training. Black line: network trained with specific stimuli, red line: network trained with no specific stimuli but only unspecific noise input. Sparseness is measured as kurtosis of the connection matrices. Note that the plateau is slightly higher for the network that was trained with a specific stimulus set. $\mathbf{f}$ Averaged input into each cell. Blue line: network before training, green line: network after training. Note that although the overall excitation is reduced after training, excitatory connection strengths to inhibitory cells (index 800-1,000) are strengthened

Thus, the overall connectivity patterns seems to be established by spike-timing-dependent plasticity. However, these two training protocols yielded slightly different levels of sparseness for the two networks. The sparseness of the networks was quantified by the kurtosis $g$ of the distribution of synaptic weights. High kurtosis corresponds to a situation with relatively few extreme connections and most other connections close to the average. Figure $3 \mathrm{e}$ displays the evolution of network sparseness during training. The initial network had a relatively low kurtosis. During learning, the kurtosis rapidly increased during the first 2 or $3 \mathrm{~h}$ and then fluctuated around a relatively stable plateau. This plateau was slightly lower for the network that was trained without specific stimuli (red line in Fig. 3e) than for the network that was trained with specific stimuli (black line in Fig. 3e). 
Similar simulations were performed on a network that additionally included connections from inhibitory cells to inhibitory cells. Again, inhibitory connections were not modeled as plastic. Although learning proceeded in a similar manner in this network, the network responses looked much different. In this network, the first pulse of the response (see Fig. 2a) was increased, while all subsequent pulses were largely diminished. We decided to omit this network from the current analyses.

\subsection{Size modulation experiment}

A size modulation experiment similar to the one by Busch et al. (2004) was performed on the trained network. In that experiment, the authors observed a monotonic increase of early gamma band activity with increasing stimulus size. This increase in bottom up salience seems to be predominantly due to increased phase-locking of the responses to the stimulus (see also Fründ et al. 2007b). We simulated different stimulus sizes by injecting current into different numbers of cells. During the experiment, learning was disabled.

Figure 4 shows time-frequency representations of network activity in response to different simulated stimulus sizes. In part (a), we see that evoked activity after stimulation with a small ( 20 cells) stimulus is virtually absent. A clear response could be observed after approximately $25 \mathrm{~ms}$ at approximately $25 \mathrm{~Hz} .{ }^{1}$ This early response increased if the stimulus size was increased to 50 cells (part b). It increased even further if the stimulus size was increased to 100 cells. Thus, the network activity displayed a similar monotonic increase of early gamma band activity with stimulus size as has been reported experimentally (Network: $F(2,36)=$ 421.85, $P<10^{-15}$, Experiment by Busch et al. (2004): $F(2,42)=11.12, P<0.001)$.

Figure $4 \mathrm{~d}$ shows a time-frequency representation of the PLF in response to a small (20 cells) stimulus. The early response that we saw in Fig. 4a is present here, too. Although the increases of the PLF were less focused in both, time and frequency, we can clearly observe a monotonic increase of this statistic with increasing stimulus size (Fig. 4e,f). Thus at least part of the modulation of the response observed in Fig. 4a-c resulted from increased phase-locking to the stimulus. In contrast, if we consider the averaged amplitudes of average membrane potential that are shown in Fig. $4 \mathrm{~g}-\mathrm{i}$, the early response was very weak. There was still a small early response, which was modulated by stimulus size. However, the strongest modulation of the simulated LFP was found considerably later, after approximately $200 \mathrm{~ms}$ at a frequency of approximately $60 \mathrm{~Hz}$. Strong and consistent modulations

\footnotetext{
${ }^{1}$ Note that the seemingly strong activity at the borders of the analyzed window is an artifact of the time-frequency analysis which becomes wider at lower frequencies.
}

of early evoked responses were observed in all 13 simulation runs. This is illustrated by the very small standard errors of the responses shown in Fig. 5a.

Figure 5a also shows results from a network in which the network connection strengths were shuffled within all excitatory connections and within all inhibitory connections (dashed lines). Thus for this network, the mean and the standard deviation of the connection strengths as well as the number of connections were all the same as for the original network. However, the fine structure of the connections was completely abolished. Figure 5a demonstrates that evoked gamma band responses are much reduced if this fine structure is disrupted. Consequently, the size manipulation has clearly weaker effects in this modified network.

We determined the firing rates of those cells that were directly stimulated separately from the firing rates of the remaining excitatory cells and the inhibitory cells. In general, excitatory cells that were not directly stimulated displayed only weak responses. In contrast, prominent responses were observed for those cells that were directly stimulated by the input as well as for the inhibitory subpopulation. We tested whether the stimulated cells were driving the inhibitory subpopulation by estimating the rate with which the responses of the stimulated cells peaked before the responses of the inhibitory cells peaked. It should be noted that this does not necessarily have to be the case as both populations also displayed spontaneous fluctuations in their activity. Thus, if the responses of the inhibitory populations were merely due to spontaneous fluctuations, the responses should peak equally often before the excited cells as after the excited cells. However, this was not the case. For the small stimulus, the cell population that was directly stimulated peaked in $88 \%$ of the trials before the inhibitory population's response peaked. This clearly suggests a driving impact of the directly stimulated cells on the inhibitory population. For larger stimulus sizes, the fraction increased to $98 \%$ for the largest stimulus. Thus, after stimulation, the inhibitory population was driven by the stimulated cells and this driving influence was stronger for larger stimuli.

\subsection{Memory experiment}

A second experiment was performed with the trained network. This experiment resembled the one by Herrmann et al. (2004a). In that experiment, the authors presented their participants images of known and unknown objects. The early gamma band responses to known objects were stronger than those to unknown objects. We repeated a similar experiment with the trained network. We considered those stimuli "known" that were presented during the training phase. In addition, we presented stimuli of the same size that were not presented during the training phase. These stimuli were 
Fig. 4 Size modulation of early gamma band responses. Left column: time-frequency representations of evoked activity for small (20 cells, a), medium (50 cells, b), and large (100 cells, c) stimuli. Middle column: time-frequency representations of the phase-locking factor for small (20 cells, d), medium (50 cells, e), and large (100 cells, f) stimuli. Right column: time-frequency representations of average amplitude for small (20 cells, $\mathbf{g}$ ), medium (50 cells, h), and large (100 cells, i) stimuli. Note, that the early evoked response at approximately $25 \mathrm{~ms}$ and $25 \mathrm{~Hz}$ $(\mathbf{a}, \mathbf{b}, \mathbf{c})$ can mainly be related to increases in the phase-locking factor (d,e,f). Stimulus intensity was $I=3.5$ during training as well as during testing (a) evoked, small

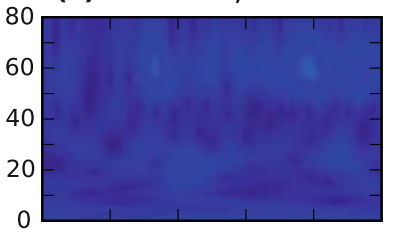

(b) evoked, medium

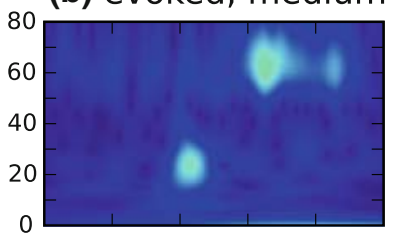

(c) evoked, large

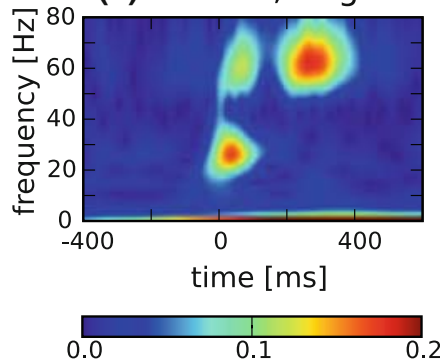

(d) PLF, small

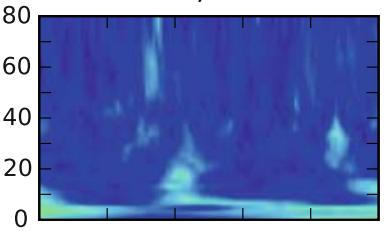

(e) PLF, medium

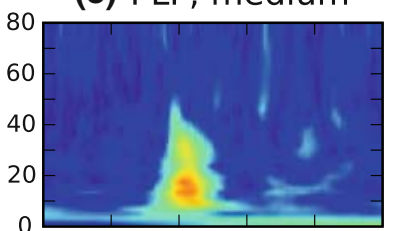

(f) PLF, large

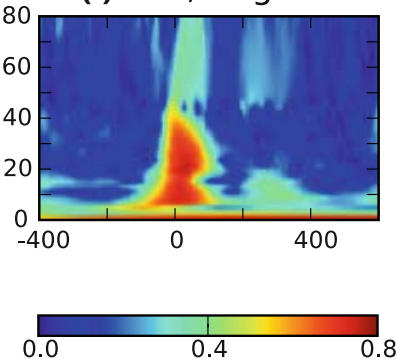

(g) amplitudes, small

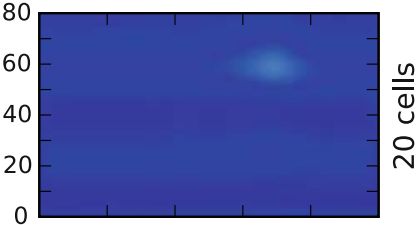

(h) amplitudes, medium

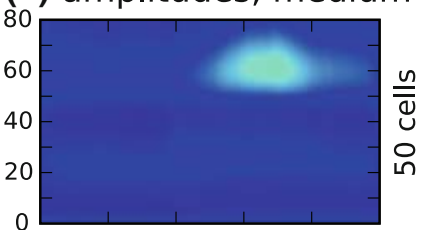

(i) amplitudes, large

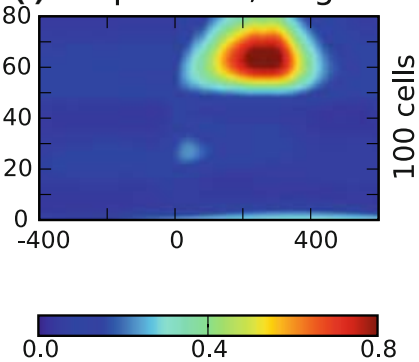

considered "unknown". During the experiment, learning was disabled.

Figure 6 displays time-frequency representations of the simulated LFP for known and unknown stimuli. When stimulated with a known stimulus, the network displayed a clear evoked response at approximately $25 \mathrm{~Hz}$ after approximately $25 \mathrm{~ms}$ (Fig. 6a). When stimulated with an unknown stimulus, this response was attenuated but still focused in time and frequency (Fig. 6b). This is similar to effects reported from experiments (Network: $F(1,24)=9.46, P<0.01$, experiment by Fründ et al. 2008a, $F(1,16)=5.24, P<0.05)$. A similar analysis for the PLF is plotted in Fig. 6c, d. This response was less focused in time and frequency than it was for the evoked activity. For averaged amplitudes, there was a very focused response with a similar time-frequency localization as for the evoked activity. Although this response was focused for known stimuli (Fig. 6e) as well as unknown stimuli (Fig. 6f), it was clearly attenuated for unknown stimuli. For the average amplitudes, there was a second, later response component at a frequency of approximately $60 \mathrm{~Hz}$. This response was visible for both, the known stimuli (Fig. 6e) and the unknown stimuli (Fig. 6f). However, after 100-200 ms, it was stronger for the known stimuli than for the unknown stimuli.

In Fig. 5b, we also show time courses of the evoked gamma band responses to known and unknown stimuli for a network in which the network connections were shuffled after the network was trained (dashed lines). Similar to the observations reported in Sect. 3.2, evoked gamma band responses are diminished in this network. Furthermore, the network does not exhibit any memory effects anymore.

\subsection{Origin of evoked and induced responses}

So far, we observed two types of responses in the model.

1. An early response at a frequency of approximately $25 \mathrm{~Hz}$ which is strongly phase locked to the onset of a stimulus.

2. A later response at a considerably higher frequency $(\approx 60 \mathrm{~Hz})$ which was only weakly or not at all phase locked to the onset of a stimulus.

We tried to identify the cells that contributed to each of these responses. To do so, we derived a pattern for each of the responses and determined the match of this pattern to the response of every single cell in every single trial. If this match was high, we concluded, that the respective cell had a large contribution to the respective response component.

The evoked response was most prominent in time-frequency representations of the event related potential, i.e., the average across all trials. Thus, we limited the bandwidth of the event-related potential time course to frequencies between 18 and $32 \mathrm{~Hz}$. The time course of this signal in 


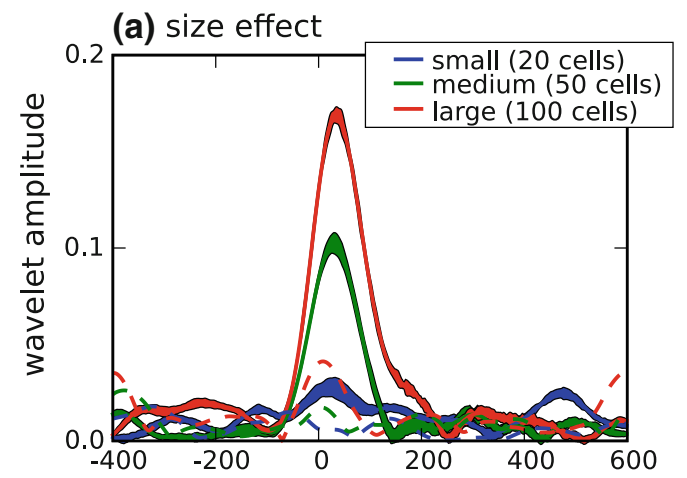

(b) memory effect

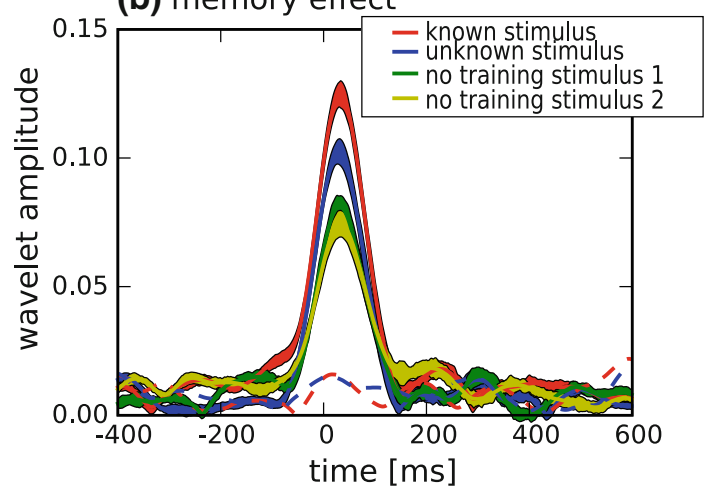

Fig. 5 Time courses of evoked activity at $25 \mathrm{~Hz}$. Solid lines indicate averages over 13 simulation runs, shaded regions indicate $95 \%$ confidence intervals of mean. a Network responses to stimuli of difference sizes. Blue lines and shading indicate responses to small stimuli (20 cells). Green lines and shading indicate responses to medium size stimuli (50 cells). Red lines and shading indicate responses to large stimuli (100 cells). b Network responses to known and unknown stimuli. Red lines and shading indicate responses to known stimuli. Blue lines and shading indicate responses to unknown stimuli. Green and yellow lines and shading correspond to the same stimuli but in a network that did not receive any specific stimulation during the learning phase. Green indicates the same stimulus as the known stimulus, yellow indicates the same stimulus as the unknown stimulus. In both parts, the dashed lines mark the same conditions as the solid lines with one difference: in these conditions, the network connections of the trained network were shuffled between all excitatory connections and between all inhibitory connections prior to experimental testing. Note that size effects and gamma band responses are diminished in these cases. Confidence intervals have roughly the same width as for the intact network

the time range from $25 \mathrm{~ms}$ before stimulus onset to $100 \mathrm{~ms}$ after stimulus onset served as the pattern for the evoked response. The time and frequency range that correspond to this pattern are marked by a green box in Fig. 7c. The pattern itself is superimposed on the event-related potential time course in Fig. 7a. For each trial and each cell, we calculated the correlation between this pattern and the time course of the membrane potential of this cell on that particular trial. Histograms of these correlations are color coded for each cell in Fig. 7b. The cells that directly received a stimulus are marked by a white bar at the bottom of Fig. 7b. Although the correlation was larger than zero for most excitatory cells; this effect was most prominent for those cells that were directly stimulated. Another point that is worth noting is that the distribution of correlations was more spread out for inhibitory cells than for excitatory cells. This indicates that for inhibitory cells, more strongly positive and more strongly negative correlated cells are present in the network.

The later gamma band response, was most prominent in time-frequency representations of the average amplitude across trials. This indicated that the phase of the contributions to this response varies from trial to trial, although the timing as well as the frequency range remain relatively constant. It is therefore not possible to quantify the contributions of single cells to this response as correlations with a single pattern. However, a necessary condition for the contribution of a particular cell is easily formulated: if a cell contributes to the late increase in amplitudes around $60 \mathrm{~Hz}$, it has to contribute to the simulated LFP on each single trial in the same time and frequency range. Thus, we limited the bandwidth of the simulated LFP on each single trial and selected the time course from 100 to $500 \mathrm{~ms}$ as the pattern for that particular trial. For each trial, we constructed a pattern for the late gamma band response and calculated the correlation between this pattern and the time course of the membrane potential of each single cell on that trial. This resulted in one correlation for each cell and each trial. The time and frequency ranges that were used to derive the patterns are marked as a red box in Fig. 7e. The average envelope of all patterns is marked as a red line in Fig. 7a. Histograms of the correlations are color coded for each cell in Fig. 7d. In contrast to the evoked response, the cells that display high contributions to the late gamma band response are not restricted to the cells that were directly stimulated. The correlations for the single cells are skewed toward positive correlations for the whole excitatory population. For the inhibitory population, the correlations spread less than in the case with the evoked gamma band response.

We performed the same experiments on a network in which all the connections were randomized. In this network, none of the reported results could be observed. Thus, STDP seems to be relevant for generating and maintaining a network state that is characterized by event-related gamma band responses.

\section{Discussion}

We presented data from a simple spiking network model, similar to the one by Izhikevich (2006). During a training phase, the network connections were modified using spiketiming-dependent plasticity. During this phase, the network was stimulated with two different input patterns. The overall network structure became more sparse, while local features of the network remained the same. After training, we conducted two experiments on the trained network. In the first 
(a) evoked, known

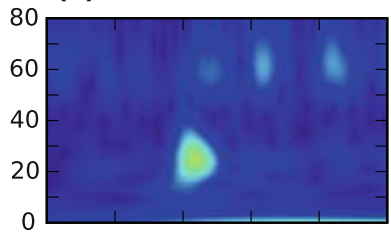

(b) evoked, unknown

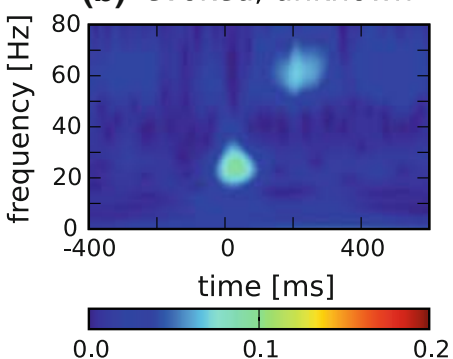

(c) PLF, known

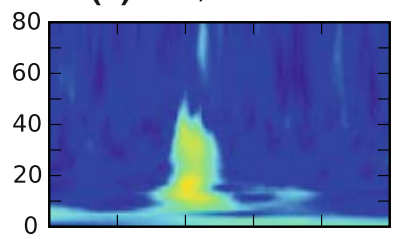

(d) PLF, unknown

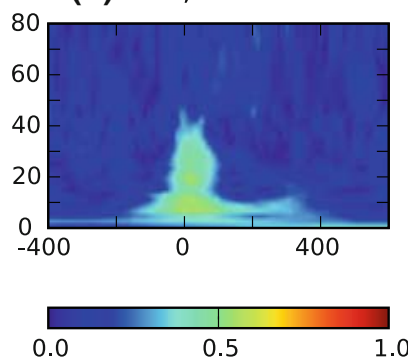

(e) amplitudes, known

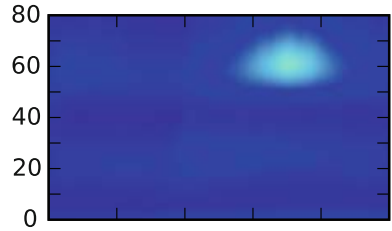

(f) amplitudes, unknown

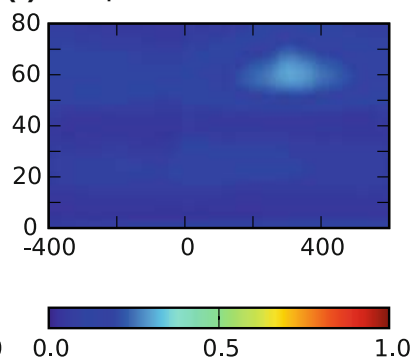

Fig. 6 Memory modulations of early gamma band responses. Left column: time-frequency representation of evoked activity for known (a) and unknown (b) stimuli. Middle column: time-frequency representation of the phase-locking factor for known (c) and unknown (d) stimuli.
Right column: time-frequency representation of average amplitude for known (e) and unknown (f) stimuli. Note that the differences that can be observed for evoked activity are virtually absent for the phase-locking factor $(\mathbf{c}, \mathbf{d})$ but very pronounced for the average amplitude $(\mathbf{e}, \mathbf{f})$ experiment, we manipulated the size of the input population. Similar to an experiment by Busch et al. (2004), we observed a monotonic increase in early gamma band responses with increasing input size. In the second experiment, we compared the responses to known stimuli, that had been presented during the training phase, and unknown stimuli that had not been presented during the training phase. Similar to an experiment by Herrmann et al. (2004a), we observed stronger early gamma band responses for known stimuli. This indicates that spike-timing-dependent plasticity drives a neural network into a state that is characterized by early gamma band responses.

Classical approaches that modeled the electroencephalogram have usually focused their attention on the interrelation of average properties (David and Friston 2003; Freeman 1975; Jansen and Rit 1995; Jirsa and Haken 1997; Nunez and Srinivasan 2006; Robinson et al. 1997, 2001; Wright and Liley 1995). Such mass models are much better in dealing with the huge number of neurons that are involved in generating the electroencephalogram than spike-based approaches such as the one employed here. Furthermore, such models provide better opportunity of fitting to real electrophysiological data in a quantitative way (David et al. 2006; Robinson et al. 2001, 2004). Mass models have provided reasonable explanations for phenomena that arise in the limit of infinitely many neurons, such as wave-like information transmission (Freeman 1975; Jirsa and Haken 1997; Nunez and Srinivasan 2006; Robinson et al. 1997), or the emergence of large-scale synchronous rhythms (Freeman 1975; Jansen and Rit 1995; Robinson et al. 1998; Rennie et al. 2000). How- ever, large-scale dynamical states are characterized not only by average firing rate but also by the variability of spiking across cells (Brunel 2000). This variability is discarded by simply considering average quantities. However, it is crucial for spike-timing-dependent plasticity. Although the analysis of the current report focused on average quantities, the simulation did not. Thus, the simulation can reasonably capture spike-timing-dependent plasticity as present in the fine structure of spiking variability.

The manipulations we performed on the model resulted in changes of early gamma band responses. These changes manifested themselves as changes in phase-locking to the onset of the stimulus. These findings have parallels in the literature on early gamma band responses. Reliable increases in phase-locking have been reported for large stimuli (Fründ et al. 2007b). However, such phase-locking does not seem to be restricted to size manipulations. Increased phase-locking has also been found in response to stimuli with high visual contrast (Schadow et al. 2007b), to stimuli with low spatial frequency (Fründ et al. 2007a), or to intense auditory stimulation (Schadow et al. 2007a). This seems to indicate that bold, salient stimuli in general result in increased phase-locking of the early gamma band response to the stimulus. For the current simulation, different levels of bottom-up saliency translate into different numbers of neurons directly stimulated. For top-down modulations of early gamma band responses, there is less clear evidence that favors one or the other property of the evoked gamma band response. Attentional modulation of early gamma band responses seems to become manifest in the amplitudes of early gamma band responses (Busch 


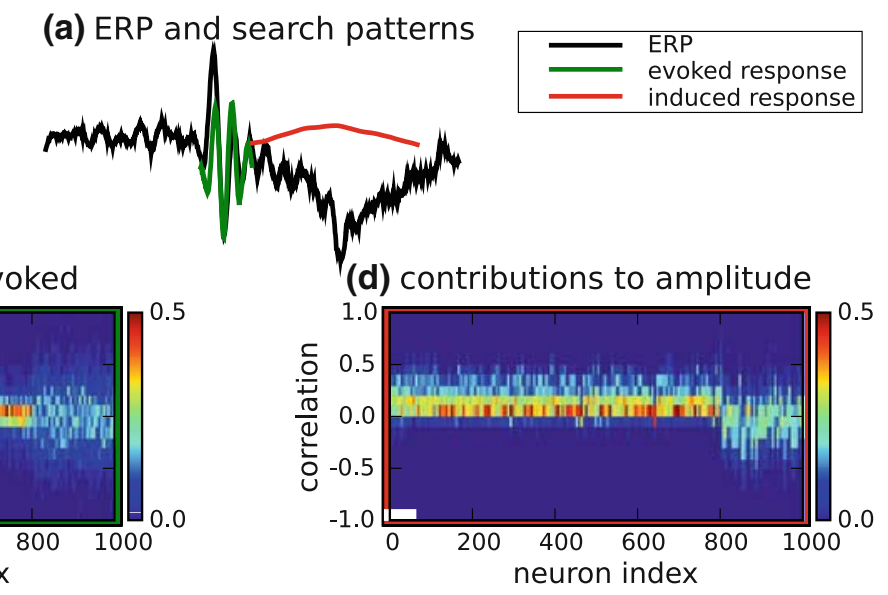

(c) evoked population activity

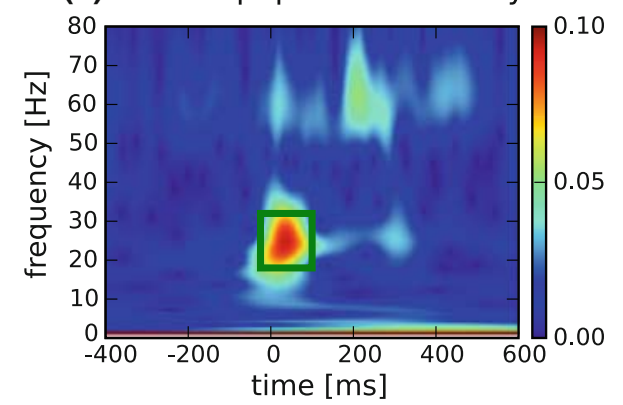

Fig. 7 Single-cell contributions to different types of response. a Simulated event-related potential (black line) and the two patterns that were searched. Green line: pattern for evoked gamma band activity. Red line: average envelope of the patterns for induced gamma band activity. b Contributions of each neuron to the evoked gamma band response. The time course of each neuron in each single trial was correlated with the pattern for the evoked gamma band response (green line in part a). Thus, for each trial and each neuron, a correlation coefficient was determined that described the correlation between the neurons activity and the evoked gamma band response. Color codes the probability for a given neuron to display the specific correlation on a given trial. A white bar at the bottom left marks the cells that received direct current input. c Time frequency representation of the evoked activity. Green box: timefrequency window of the pattern for the evoked gamma band response.

et al. 2006b). However, another study only observed familiarity effects for the phase-locking of the early gamma band response but not for average amplitude (Fründ et al. 2008a). Thus, the EEG data are not conclusive in this respect.

Spike-timing-dependent plasticity drives the network into a state that is characterized by evoked gamma band responses. Although it had previously been demonstrated that spike-timing-dependent plasticity can stabilize global network states (e.g., Song et al. 2000), it is interesting to note, that evoked gamma band responses are a characteristic part of this. Furthermore, the eigenvalue analysis suggests that the emergence of evoked gamma band activity proceeds relatively independent of the decay of the low-frequency components of the network activity. This is a prediction that has, to our knowledge not yet been tested experimentally.

\section{(e) amplitude population activity}

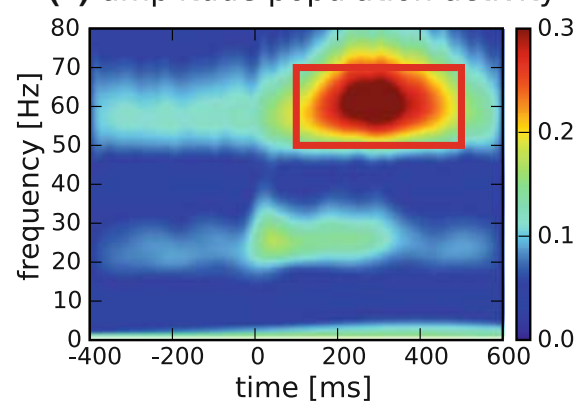

d Contributions of each neuron to the late amplitude changes in the gamma band. The time course of each neuron in each single trial was correlated with the band limited simulated LFP on that trial (the average envelope of these population activities is indicated as a red line in part a). Thus, for each trial and each neuron, a correlation coefficient was determined that described the correlation between the neurons activity and the late gamma band activity on that trial. The average of these late gamma band activities across trials is the late gamma band response (marked by a red box in part e). Color codes the probability for a given neuron to display the specific correlation on a given trial. A white bar at the bottom left marks the cells that received direct current input. e Time frequency representation of the average amplitudes of population activity. Red box: time-frequency window of the patterns for the late gamma band response

A very prominent phenomenon associated with scalp measured gamma band activity are so-called induced gamma band responses (for review see Basar-Eroglu et al. 1996; Tallon-Baudry and Bertrand 1999). There has recently been some doubt as to whether such responses could be measured from scalp EEG at all (Yuval-Greenberg et al. 2008). Interestingly, our model displays induced gamma band responses as indicated by our amplitude measure. These responses are more pronounced for known stimuli as compared to unknown stimuli, which is exactly what many studies of scalp measured gamma band responses found (Busch et al. 2006a; Gruber and Müller 2005, 2006; Lachaux et al. 2005; TallonBaudry et al. 1997, see Herrmann et al. 2004b for a review). In addition, this response was modulated by the size of the input stimuli in a similar way as recently observed for 
natural images (Fründ et al. 2008b). However, the response frequencies observed in our model are relatively high (approximately $50-70 \mathrm{~Hz}$ ) for scalp measured induced gamma band responses. Thus, it is unclear in how far the later modulations of average amplitude can be related to the induced gamma band responses reported in the literature. If they could, the fact that they emerge directly from the spiketiming-dependent plasticity learning rule suggests that these brain responses are related to very basal processing.

In the present simulation, we observed stronger evoked responses for (i) larger stimuli and (ii) for known as compared to unknown stimuli. The frequency of these responses is in the $\beta / \gamma$ frequency range $(\approx 20-90 \mathrm{~Hz})$. Previous studies have related gamma band activity to activity of the inhibitory subpopulation (Izhikevich 2006) or to recurrent activity within the inhibitory subpopulation (see Whittington et al. 2000, for a review). The current model does not have recurrent connections within the inhibitory subpopulation. Thus, a purely inhibitory origin of the gamma band activity in this model is not possible. ${ }^{2}$ The correlation analysis in Sect. 3.4 suggests two different origins for the early and late response. The early gamma band response was predominantly input driven. The excitatory cells that strongly contribute to this response component are those cells that were directly excited by the stimulus. The late gamma band response was not related to direct driving from the input. This is indicated by the fact, that all excitatory cells contributed more or less equally strong to the late gamma band response. This suggests that the late gamma band response emerges from the interactions within the whole network. A similar effect, albeit on a different scale has been reported for scalp measured EEG, too. The evoked gamma band response is typically restricted to very few scalp locations, while the late gamma band response is much more widespread (e.g., Busch et al. 2006a; Fründ et al. 2008a). However, such comparisons have to remain very speculative.

We conclude, that very simple random networks under the influence of spike-timing-dependent plasticity can reproduce findings from experiments with humans. It is plausible that early gamma band responses in humans are shaped by bottom-up processing and by low level learning processes such as spike-timing-dependent plasticity. Furthermore, the model predicts that later gamma band responses are an emergent network property.

Acknowledgments The authors would like to thank Eugene M. Izhikevich for helpful comments on an earlier version of the manuscript and the suggestion to use his code for these simulations. This work was

\footnotetext{
2 A similar model that included inhibitory projections to inhibitory cells did even show weaker gamma band activity. In this model the differences due to stimulus size and memory were comparable to the present one.
}

supported by the Deutsche Forschungsgemeinschaft (DFG, HE 3353/ 6-1) and the Bundesministerium für Bildung und Forschung (BMBF, Bernstein Gruppe Magdeburg).

Open Access This article is distributed under the terms of the Creative Commons Attribution Noncommercial License which permits any noncommercial use, distribution, and reproduction in any medium, provided the original author(s) and source are credited.

\section{References}

Abeles M (1991) Corticonics. Cambridge Universtity Press, New York Arieli A, Sterkin A, Grinvald A, Aertsen A (1996) Dynamics of ongoing activity: explanation of the large variability in evoked cortical responses. Science 273(5283):1868-1871

Başar E (1980) EEG-brain dynamics. Elsevier, Amsterdam

Basar-Eroglu C, Struber D, Schurmann M, Stadler M, Basar E (1996) Gamma-band responses in the brain: a short review of psychophysiological correlates and functional significance. Int J Psychophysiol 24(1-2):101-112

Berger H (1929) Über das Elektrenkephalogramm des Menschen. Arch Psychiatr 87:528-570

Bi GQ, Poo MM (1998) Synaptic modifications in cultured hippocampal neurons: dependence on spike timing, synaptic strength, and postsynaptic cell type. J Neurosci 18(24):10464-10472

Brunel N (2000) Dynamics of sparsely connected networks of excitatory and inhibitory spiking neurons. J Comput Neurosci 8:183208

Busch NA, Debener S, Kranczioch C, Engel AK, Herrmann CS (2004) Size matters: effects of stimulus size, duration and eccentricity on the visual gamma-band response. Clin Neurophysiol 115(8): $1810-1820$

Busch NA, Herrmann CS, Müller MM, Lenz D, Gruber T (2006a) A cross-laboratory study of event-related gamma activity in a standard object recognition paradigm. NeuroImage 33:1169-1177

Busch NA, Schadow J, Fründ I, Herrmann CS (2006b) Time-frequency analysis of target detection reveals an early interface between bottom-up and top-down processes in the gamma-band. NeuroImage 29(4):1106-1116

Connors BW, Gutnick MJ (1990) Intrinsic firing patterns of diverse neocortical neurons. Trends Neurosci 13(3):99-104

David O, Friston KJ (2003) A neural mass model for MEG/EEG: coupling and neuronal dynamics. NeuroImage 20:1743-1755

David O, Kiebel SJ, Harrison LM, Mattout J, Kilner JM, Friston KJ (2006) Dynamic causal modeling of evoked responses in EEG and MEG. NeuroImage 30(4):1255-1272

Debener S, Herrmann CS, Kranczioch C, Gembris D, Engel AK (2003) Top-down attentional processing enhances auditory evoked gamma band activity. NeuroReport 14(5):683-686

Fisher N (1993) Statistical analysis of circular data. Cambridge Universtity Press, New York

Fox MD, Snyder AZ, Vincent JL, Raichle ME (2007) Intrinsic fluctuations within cortical systems account for intertrial variability in human behavior. Neuron 56(1):171-184

Freeman WJ (1975) Mass action in the nervous system. Academic Press, New York

Freeman WJ (2000) Neurodynamics. Springer, London

Fründ I, Busch NA, Körner U, Schadow J, Herrmann CS (2007a) EEG oscillations in the gamma and alpha range respond differently to spatial frequency. Vis Res 47(15):2086-2098

Fründ I, Schadow J, Busch NA, Körner U, Herrmann CS (2007b) Evoked gamma oscillations in human scalp EEG are testretest reliable. Clin Neurophysiol 118(1):221-227 
Fründ I, Busch NA, Schadow J, Gruber T, Körner U, Herrmann CS (2008a) Time pressure modulates electrophysiological correlates of early visual processing. PLoS One 3(2):e1675

Fründ I, Schadow J, Busch NA, Naue N, Körner U, Herrmann CS (2008b) Anticipation of natural stimuli modulates EEG dynamics: physiology and simulation. Cogn Neurodyn 2(2):89-100

Gruber T, Müller MM (2005) Oscillatory brain activity dissociates between associative stimulus content in a repetition priming task in the human EEG. Cereb Cortex 15(1):109-116

Gruber T, Müller MM (2006) Oscillatory brain activity in the human EEG during indirect and direct memory tasks. Brain Res 1097(1):194-204

Hanslmayr S, Klimesch W, Sauseng P, Gruber W, Doppelmayr M, Freunberger R, Pecherstorfer T, Birbaumer N (2007) Alpha phase reset contributes to the generation of ERPs. Cereb Cortex 17(1):18

Herrmann CS, Lenz D, Junge S, Busch NA, Maess B (2004a) Memorymatches evoke human gamma-responses. BMC Neurosci 5(13)

Herrmann CS, Munk MH, Engel AK (2004b) Cognitive functions of gamma-band activity: memory match and utilization. Trends Cogn Sci 8(8):347-355

Izhikevich EM (2003) Simple model of spiking neurons. IEEE Trans Neural Netw 14(6):1569-1572

Izhikevich EM (2006) Polychronization: computation with spikes. Neural Comput 18:245-282

Jansen BH, Rit VG (1995) Electroencephalogram and visual evoked potential generation in a mathematical model of coupled cortical columns. Biol Cybern 73:357-366

Jirsa VK, Haken H (1997) A derivation of a macroscopic field theory of the brain from the quasi-microscopic neural dynamics. Phys D 99:503-526

Karakaş S, Başar E (1998) Early gamma response is sensory in origin: a conclusion based on cross-comparison of results from multiple experimental paradigms. Int J Psychophysiol 31:13-31

Kenet T, Bibitchkov D, Tsodyks M, Grinvald A, Arieli A (2003) Spontaneously emerging cortical representations of visual attributes. Nature 425:954-956

Lachaux JP, George N, Tallon-Baudry C, Martinerie J, Hugueville L, Minotti L, Kahane P, Renault B (2005) The many faces of the gamma band response to complex visual stimuli. NeuroImage 25:491-501

Makeig S, Westerfield M, Jung TP, Enghoff S, Townsend J, Courchesne E, Sejnowski TJ (2002) Dynamic brain sources of visual evoked responses. Science 295:690-694

Markram H, Lübke J, Frotscher M, Sakmann B (1997) Regulation of synaptic efficacy by coincidence of postsynaptic APs and EPSPs. Science 275:213-215

Morup M, Hansen LK, Herrmann CS, Parnas J, Arnfred SM (2006) Parallel factor analysis as an exploratory tool for wavelet transformed event-related EEG. NeuroImage 29(3):938-947

Nunez PL, Srinivasan R (2006) Electric fields of the brain, 2nd edn. Oxford University Press, New York
Rennie CJ, Wright JJ, Robinson PA (2000) Mechanisms of cortical electrical activity and emergence of gamma rhythm. J Theor Biol 205:17-35

Robinson PA, Rennie CJ, Wright JJ (1997) Propagation and stability of waves of electrical activity in the cerebral cortex. Phys Rev E 56(1):826-840

Robinson PA, Wright JJ, Rennie CJ (1998) Synchronous oscillations in the cerebral cortex. Phys Rev E 57(4):4578-4588

Robinson PA, Rennie CJ, Wright JJ, Bahramali H, Gordon E, Rowe DL (2001) Prediction of electroencephalographic spectra from neurophysiology. Phys Rev E 63(2):021, 903

Robinson PA, Rennie CJ, Rowe DL, O'Connor SC (2004) Estimation of multiscale neurophysiologic parameters by electroencephalographic means. Hum Brain Mapp 25:53-72

Schadow J, Lenz D, Thaerig S, Busch NA, Fründ I, Herrmann CS (2007a) Stimulus intensity affects early sensory processing: sound intensity modulates auditory evoked gamma-band activity in human EEG. Int J Psychophysiol 65(2):152-161

Schadow J, Lenz D, Thaerig S, Busch NA, Fründ I, Rieger JW, Herrmann CS (2007b) Stimulus intensity affects early sensory processing: visual contrast modulates evoked gamma-band activity in human EEG. Int J Psychophysiol 66(1):28-36

Song S, Miller KD, Abbott LF (2000) Competitive Hebbian learning through spike-timing dependent synaptic plasticity. Nat Neurosci 3(9):919-926

Tallon-Baudry C, Bertrand O (1999) Oscillatory gamma activity in humans and its role in object representation. Trends Cogn Sci 3(4):151-162

Tallon-Baudry C, Bertrand O, Delpuech C, Pernier J (1996) Stimulus specificity of phase-locked and on-phase-locked $40 \mathrm{~Hz}$ visual responses in human. J Neurosci 16(13):4240-4249

Tallon-Baudry C, Bertrand O, Delpuech C, Pernier J (1997) Oscillatory $\gamma$-band (30-70 hz) activity induced by a visual search task in humans. J Neurosci 17(2):722-734

Tiitinen H, Sinkkonen J, Reinikainen K, Alho K, Lavikainen J, Näätänen R (1993) Selective attention enhances the auditory 40$\mathrm{Hz}$ transient response in humans. Nature 364:59-60

Tsodyks M, Kenet T, Grinvald A, Arieli A (1999) Linking spontaneous activity of single cortical neurons and the underlying functional architecture. Science 286(1943):1943-1946

Whittington MA, Traub RD, Kopell N, Ermentrout B, Buhl EH (2000) Inhibition-based rhythms: experimental and mathematical observations on network dynamics. Int J Psychophysiol 38:315336

Wright JJ, Liley DTJ (1995) Simulation of electrocortical waves. Biol Cybern 72(4):347-356

Yuval-Greenberg S, Tomer O, Keren AS, Nelken I, Deouell LY (2008) Transient induced gamma-band response in EEG as a manifestation of miniature saccades. Neuron 58(3):429-441 